\title{
Quasiconvex Semidefinite Minimization Problem
}

\author{
R. Enkhbat ${ }^{1}$ and T. Bayartugs ${ }^{2}$ \\ ${ }^{1}$ National University of Mongolia, Mongolia \\ ${ }^{2}$ Mongolian University of Science and Technology, Mongolia \\ Correspondence should be addressed to R. Enkhbat; renkhbat46@yahoo.com
}

Received 23 May 2013; Accepted 7 November 2013

Academic Editor: Jein-Shan Chen

Copyright (C) 2013 R. Enkhbat and T. Bayartugs. This is an open access article distributed under the Creative Commons Attribution License, which permits unrestricted use, distribution, and reproduction in any medium, provided the original work is properly cited.

We introduce so-called semidefinite quasiconvex minimization problem. We derive new global optimality conditions for the above problem. Based on the global optimality conditions, we construct an algorithm which generates a sequence of local minimizers which converge to a global solution.

\section{Introduction}

Semidefinite linear programming can be regarded as an extension of linear programming and solves the following problem:

$$
\begin{gathered}
\min \langle C, X\rangle_{F}, \\
\left\langle A_{j}, X\right\rangle_{F} \leqslant b_{j}, \quad j=1,2, \ldots, s, \\
X \geqslant 0,
\end{gathered}
$$

where $X \in \mathbb{R}^{n \times n}$ is a matrix of variables and $A_{j} \in \mathbb{R}^{n \times n}, j=$ $1,2, \ldots, s . X \geqslant 0$ is notation for " $X$ is positive semidefinite". $\langle\cdot, \cdot\rangle_{F}$ denotes Frobenius norm and $\|X\|_{F}=\sqrt{\langle A, A\rangle_{F}}$.

Semidefinite programming finds many applications in engineering and optimization [1]. Most interior-point methods for linear programming have been generalized to semidefinite convex programming [1-3]. There are many works devoted to the semidefinite convex programming problem but less attention so for has been paid to quasiconvex programming semidefinite quasiconvex minimization problem.

The aim of this paper is to develop theory and algorithms for the semidefinite quasiconvex programming. The paper is organized as follows. Section 2 is devoted to formulation of semidefinite quasiconvex programming and its global optimality conditions. In Section 3, we consider an approximation of the level set of the objective function and its properties.

\section{Problem Definition and Optimality Conditions}

Let $X$ be matrices in $\mathbb{R}^{n \times n}$, and define a scalar matrix function as follows:

$$
f: \mathbb{R}^{n \times n} \longrightarrow \mathbb{R}
$$

Definition 1. Let $f(X)$ be a differentiable function of the matrix $X$. Then

$$
f^{\prime}(X)=\left(\frac{\partial f(X)}{\partial x_{i j}}\right)_{n \times n} .
$$

Introduce the Frobenius scalar product as follows:

$$
\langle X, Y\rangle_{F}=\sum_{i=1}^{n} \sum_{j=1}^{n} x_{i j} y_{i j}, \quad \forall X, Y \in \mathbb{R}^{n \times n} .
$$

If $f(\cdot)$ is differentiable, then it can be checked that

$$
f(X+H)-f(X)=\left\langle f^{\prime}(X), H\right\rangle_{F}+o\left(\|H\|_{F}\right) .
$$

Definition 2. A set $\mathbb{D} \subset \mathbb{R}^{n \times n}$ is convex if $\alpha X+(1-\alpha) Y \in \mathbb{D}$ for all $X, Y \in \mathbb{D}$ and $\alpha \in[0,1]$. 
Definition 3. The function $f: \mathbb{D} \rightarrow \mathbb{R}$ is said to be quasiconvex on $\mathbb{D}$ if

$$
\begin{array}{r}
f(\alpha X+(1-\alpha) Y) \leqslant \max \{f(X), f(Y)\} \\
\forall X, Y \in \mathbb{D}, \quad \alpha \in[0,1] .
\end{array}
$$

The well-known property of a convex function [3] can be easily generalized as follows.

Lemma 4. A function $f: R^{n \times n} \rightarrow R$ is quasiconvex if and only if the set

$$
L_{c}(f)=\left\{X \in R^{n \times n} \mid f(X) \leqslant c\right\}
$$

is convex for all $c \in R$.

Proof

Necessity. Suppose that $c \in R$ is an arbitrary number and $X, Y \in L_{c}(f)$. By the definition of quasiconvexity, we have

$$
\begin{aligned}
f(\alpha X+(1-\alpha) Y) & \leqslant \max \{f(X), f(Y)\} \\
& \leqslant c \quad \forall \alpha \in[0,1],
\end{aligned}
$$

which means that the set $L_{c}(f)$ is convex.

Sufficiency. Let $L_{c}(f)$ be a convex set for all $c \in R$. For arbitrary $X, Y \in R^{n}$, define $c^{o}=\max \{f(X), f(Y)\}$. Then $X \in L_{c^{o}}(f)$ and $Y \in L_{c^{o}}(f)$. Consequently, $\alpha X+(1-\alpha Y) \epsilon$ $L_{c^{o}}(f)$, for any $\alpha \in[0,1]$. This completes the proof.

Lemma 5. Let $f: \mathbb{R}^{n \times n} \rightarrow \mathbb{R}$ be a quasiconvex and differentiable function. Then the inequality $f(X) \leqslant f(Y)$ for $X, Y \in \mathbb{R}^{n \times n}$ implies that

$$
\left\langle f^{\prime}(Y), X-Y\right\rangle_{F} \leqslant 0
$$

where $f^{\prime}(X)=\left(\partial f(X) / \partial x_{i j}\right)_{n \times n}$ and $\langle\cdot, \cdot\rangle_{F}$ denotes the Frobenius scalar product of two matrices.

Proof. Since $f$ is quasiconvex,

$$
f(\alpha X+(1-\alpha) Y) \leqslant \max \{f(X), f(Y)\}=f(Y)
$$

for all $\alpha \in[0,1]$ and $X, Y \in R^{n \times n}$ such that $f(X) \leqslant f(Y)$. By Taylor's formula, there is a neighborhood of the point $Y$ on which:

$$
\begin{aligned}
& f(Y+\alpha(X-Y))-f(Y) \\
& \quad=\alpha\left(\left\langle f^{\prime}(Y), X-Y\right\rangle_{F}+\frac{o\left(\alpha\|X-Y\|_{F}\right)}{\alpha}\right) \leqslant 0, \quad \alpha>0 .
\end{aligned}
$$

From the fact that $o\left(\alpha\|x-y\|_{F}\right) / \alpha \stackrel{\alpha \rightarrow o}{\longrightarrow} 0$, we obtain $\left\langle f^{\prime}(Y), X-Y\right\rangle_{F} \leqslant 0$ which completes the proof.
Consider the problem of minimizing a differentiable quasiconvex matrix function subject to constraints

$$
\begin{gathered}
\min f(X) \\
\text { subject to } \quad g_{j}(X) \leqslant b_{j}, \quad j=1,2, \ldots, s, \\
X \geqslant 0,
\end{gathered}
$$

where $g_{j}: \mathbb{R}^{n \times n} \rightarrow \mathbb{R}, j=1,2, \ldots, s$ are scalar functions and $X \geqslant 0$ are positive semidefinite matrices, $b_{j} \in \mathbb{R}$.

We call problem (12)-(14) as the semidefinite quasiconvex minimization problem.

Denote by $\mathbb{D}$ a constraint set of the problem as follows:

$$
\mathbb{D}=\left\{X \in \mathbb{R}^{n \times n} \mid g(X)_{j} \leqslant b_{j}, j=1,2, \ldots, s ; X \geqslant 0\right\} .
$$

Then problems (12)-(14) reduce to

$$
\min _{x \in \mathbb{D}} f(X) .
$$

In general, the set $\mathbb{D}$ is nonconvex. Problem (16) is nonconvex and belongs to a class of global optimization problems in Banach space.

We formulate a new global optimality condition for problem (16) in the following. For this purpose,we introduce the level set $E_{f(Z)}(f)$ of the function $f: \mathbb{R}^{n \times n} \rightarrow \mathbb{R}$ at a point $Z \in \mathbb{R}^{n \times n}:$

$$
E_{f(Z)}(f)=\left\{Y \in \mathbb{R}^{n \times n} \mid f(Y)=f(Z)\right\} .
$$

Then global optimality conditions for problem (16) can be formulated as follows.

Theorem 6. Let $Z$ be a solution of problem (16). Then

$$
\left\langle f^{\prime}(X), X-Y\right\rangle_{F} \geqslant 0 \quad \forall Y \in E_{f(Z)}(f), X \in D,
$$

where $E_{c}(f)=\left\{Y \in R^{n \times n} \mid f(Y)=c\right\}$. If, in addition,

$$
\lim _{\|X\| \rightarrow \infty} f(X)=+\infty, \quad f^{\prime}\left(X+\alpha f^{\prime}(X)\right) \neq 0
$$

holds for all $X \in D$ and $\alpha \geq 0$, then condition (18) becomes sufficient.

\section{Proof}

Necessity. Assume that $z$ is a solution of problem (16). Let $X \in$ $D$ and $Y \in E_{f(Z)}(f)$. Then we have $0 \geqslant f(Z)-f(X)=f(Y)-$ $f(X)$, and Lemma 5 implies $\left\langle f^{\prime}(X), X-Y\right\rangle_{F} \geqslant 0$.

Sufficiency. Suppose, on the contrary, that $Z$ is not a solution of (16). Then there exists a $U \in D$ such that $f(U)<f(Z)$. Construct a ray $Y_{\alpha}$ for $\alpha>0$ defined by

$$
Y_{\alpha}=U+\alpha f^{\prime}(U) \text {. }
$$

We claim that $f\left(Y_{\alpha}\right)>f(U)$ holds for all positive $\alpha$. By Taylor's formula, we have

$$
f\left(U+\alpha f^{\prime}(U)\right)-f(U)=\alpha\left(\left\|f^{\prime}(U)\right\|^{2}+\frac{o\left(\alpha\left\|f^{\prime}(U)\right\|\right)}{\alpha}\right)
$$


for small $\alpha>0$, where $\lim _{\alpha \rightarrow 0+} o\left(\alpha\left\|f^{\prime}(U)\right\|\right) / \alpha=0$. Therefore, there exists $\alpha_{o}>0$ such that $f\left(Y_{\alpha}\right)-f(U)>$ 0 holds for all $\alpha \in\left(0, \alpha_{o}\right)$. Hence, by Lemma 5, we have $\left\langle f^{\prime}\left(U+\alpha_{o} f^{\prime}(U)\right), f^{\prime}(U)\right\rangle_{F} \geqslant 0$ since $f^{\prime}(U) \neq 0$ and $f^{\prime}(U+$ $\left.\alpha_{o} f^{\prime}(U)\right) \neq 0$ by the assumption. Note that for all $\gamma>1$, we also have $f\left(U+\gamma \alpha_{o} f^{\prime}(U)\right)>f\left(U+\alpha_{o} f^{\prime}(U)\right)$; for otherwise, we would have $f\left(U+\gamma \alpha_{o} f^{\prime}(U)\right) \leqslant f\left(U+\alpha_{o} f^{\prime}(U)\right)$, and consequently, by Lemma $5,\left\langle f^{\prime}\left(U+\alpha_{o} f^{\prime}(U)\right), \alpha_{o}(\gamma-1) f^{\prime}(U)\right\rangle_{F} \geqslant$ 0 , which would imply $\gamma \leqslant 1$ which is contradicting to the assumption that $\gamma>1$. Moreover, we can show that $f(U+$ $\left.\gamma \alpha_{o} f^{\prime}(U)\right)$ is increasing in $\gamma>0$. If $f\left(U+\gamma^{\prime} \alpha_{o} f^{\prime}(U)\right)<$ $f\left(U+\gamma \alpha_{o} f^{\prime}(U)\right)$ holds for some $\gamma^{\prime}>\gamma$, then $\alpha_{o}\left(\gamma^{\prime}-\right.$ $\gamma)\left\langle f^{\prime}\left(U+\gamma \alpha_{o} f^{\prime}(U)\right), f^{\prime}(U)\right\rangle_{F} \leqslant 0$, which would contradict the fact that $\gamma^{\prime}>\gamma$. These prove our claim $f\left(Y_{\alpha}\right)>f(U)$ for all $\alpha>0$.

Now it is obvious that the function $\varphi: R^{+} \rightarrow R$ defined as

$$
\varphi(\alpha)=f\left(Y_{\alpha}\right)
$$

is continuous on $[0, \infty)$. Also, with assumption (19) implies $\lim _{\alpha \rightarrow \infty} \varphi(\alpha)=+\infty$, and therefore, there exists an $\widehat{\alpha}$ such that $\varphi(\widehat{\alpha})>f(Z)$. Using the continuity of $\varphi(\alpha)$ and the inequalities $\varphi(\widehat{\alpha})>f(Z)>f(U)$, there exists an $\bar{\alpha}$ such that

$$
f\left(Y+\bar{\alpha} f^{\prime}(U)\right)=f(Z),
$$

which means that $Y_{\bar{\alpha}} \in E_{f(Z)}(f)$. On the other hand, we have $f^{\prime}(U)=(1 / \bar{\alpha})\left(Y_{\bar{\alpha}}-U\right)$. Thus we get

$$
\begin{aligned}
\left\langle f^{\prime}(U), U-Y_{\bar{\alpha}}\right\rangle_{F} & =\frac{1}{\bar{\alpha}}\left\langle Y_{\bar{\alpha}}-U, U-Y_{\bar{\alpha}}\right\rangle_{F} \\
& =-\frac{1}{\bar{\alpha}}\left\|Y_{\bar{\alpha}}-Y\right\|_{F}^{2}<0,
\end{aligned}
$$

which contradicts (18). This means that $Z$ must be a solution of (16).

Example 7. Consider the following problem:

$$
\begin{gathered}
\min _{X \in D}\left(f(X)=\|X\|_{F}^{2}\right), \\
\text { subject to } \mathbb{D}=\left\{X \in \mathbb{R}^{n \times n} \mid \underline{X}=\left(\begin{array}{ll}
2 & 3 \\
4 & 1
\end{array}\right) \leqslant X \leqslant \bar{X}\right. \\
\left.=\left(\begin{array}{ll}
7 & 5 \\
8 & 9
\end{array}\right), X \geqslant 0\right\} .
\end{gathered}
$$

Example 8. Consider the fractional programming problem

$$
\min _{X \in D}\left(f(x)=\frac{f_{1}(X)}{f_{2}(X)}\right),
$$

where $f_{1}$ is convex and differentiable on $R^{n \times n}$ and $f_{2}$ is concave and differentiable on $R^{n \times n}$. Suppose that $f_{1}$ and $f_{2}$ are defined positively on a ball $B$ containing a subset $D \subset R^{n \times n}$; that is,

$$
f_{1}(X)>0, \quad f_{2}(X)>0 \quad \forall X \in D \subset B .
$$

We will call this problem as the mixed fractional minimization problem. By Lemma 4, we can easily show that $f(X)$ is quasiconvex. Hence, the optimality condition (13) at a solution $Z$ of (27) is as follows:

$$
\begin{array}{r}
\sum_{i=1}^{n} \sum_{j=1}^{n}\left(\frac{\partial f_{1}(X)}{\partial x_{i j}} f_{2}(X)-\frac{\partial f_{2}(X)}{\partial x_{i j}} f_{1}(X)\right) \frac{\left(x_{i j}-y_{i j}\right)}{f_{2}^{2}(X)} \geq 0 \\
\forall Y \in E_{f(Z)}(f), \quad X \in D .
\end{array}
$$

\section{An Algorithm for the Convex Minimization Problem}

We consider the quasiconvex minimization problem as a special case of problem (16):

$$
\min _{X \in D} f(X),
$$

where $f: R^{n \times n} \rightarrow R$ is strongly convex and continuously differentiable and $D$ is an arbitrary compact set in $R^{n \times n}$. In this case, then we can weaken condition (19) as shown in the next theorem.

Theorem 9. Let $Z$ be a solution of problem (30). Then

$$
\left\langle f^{\prime}(X), X-Y\right\rangle_{F} \geqslant 0 \quad \forall Y \in E_{f(Z)}(f), X \in D .
$$

If, in addition,

$$
\min _{X \in D}\left\|f^{\prime}(X)\right\|_{F}>0
$$

holds, then condition (31) is also sufficient.

Proof

Necessity. Assume that $z$ is a solution of problem (30). Consider $X \in D$ and $Y \in E_{f(Z)}(f)$. Then by the convexity of $f$, we have

$$
0 \geqslant f(Z)-f(X)=f(Y)-f(X) \geqslant\left\langle f^{\prime}(X), Y-X\right\rangle_{F} .
$$

Sufficiency. Let us prove the assertion by contradiction. Assume that (31) holds and there exists a point $U \in D$ such that

$$
f(U)<f(Z) .
$$

Clearly, $f^{\prime}(U) \neq 0$ by assumption (32). Now define $U_{\alpha}$ as follows for $\alpha>0$ :

$$
U_{\alpha}=U+\alpha f^{\prime}(U)
$$

Then, by the convexity of $f$, we have

$$
f\left(U_{\alpha}\right)-f(U) \geqslant\left\langle f^{\prime}(U), U_{\alpha}-U\right\rangle_{F}=\alpha\left\|f^{\prime}(U)\right\|_{F}^{2},
$$


which implies

$$
f\left(U_{\alpha}\right) \geqslant f(U)+\alpha\left\|f^{\prime}(U)\right\|_{F}^{2}>f(U) .
$$

Then find $\alpha=\bar{\alpha}$ such that

$$
f(U)+\bar{\alpha}\left\|f^{\prime}(U)\right\|_{F}^{2}=f(Z) ;
$$

that is,

$$
\bar{\alpha}=\frac{f(Z)-f(U)}{\left\|f^{\prime}(U)\right\|_{F}^{2}}>0 .
$$

Thus we get

$$
f\left(U_{\bar{\alpha}}\right) \geqslant f(U)+\bar{\alpha}\left\|f^{\prime}(U)\right\|_{F}^{2}=f(Z)>f(U) .
$$

Define a function $h: R^{+} \rightarrow R$ as

$$
h(\alpha)=f\left(U+\alpha f^{\prime}(U)\right)-f(Z),
$$

where $R^{+}=\{\alpha \in R \mid \alpha \geqslant 0\}$. It is clear that $h$ is continuous on $[0,+\infty)$. Note that $h(\bar{\alpha}) \geqslant 0$ and $h(0)<0$. There are two cases with respect to the values of $h(\bar{\alpha})$ which we should consider.

Case a. $h(\bar{\alpha})=0\left(\right.$ or $\left.f\left(U+\bar{\alpha} f^{\prime}(U)\right)=f(Z)\right)$, then

$$
\begin{aligned}
\left\langle f^{\prime}(U), U-U_{\bar{\alpha}}\right\rangle_{F} & =-\left\langle f^{\prime}(U), \bar{\alpha} f^{\prime}(U)\right\rangle_{F} \\
& =-\bar{\alpha}\left\|f^{\prime}(U)\right\|_{F}^{2}<0,
\end{aligned}
$$

contradicting condition (31).

Case b. $h(\bar{\alpha})>0$ and $h(0)<0$. Since $h$ is continuous, there exists a point $\alpha_{o} \in(0, \bar{\alpha})$ such that $h\left(\alpha_{o}\right)=0$ (or $f(U+$ $\left.\alpha_{o} f^{\prime}(U)\right)=f(Z)$ ). Then we have

$$
\left\langle f^{\prime}(U), U-U_{\alpha_{o}}\right\rangle_{F}=-\alpha_{o}\left\|f^{\prime}(U)\right\|_{F}^{2}<0,
$$

again contradicting (31).

Thus, in both cases, we find contradictions, proving the theorem.

Now using the function $P(Y)=\min _{X \in D}\left\langle f^{\prime}(X), X-Y\right\rangle_{F}$, $Y \in R^{n \times n}$, we reformulate Theorem 9 in terms of function $\psi(z)$ defined as follows:

$$
\psi(Z)=\min _{Y \in E_{f(Z)}(f)} P(Y), \quad Z \in D .
$$

Theorem 10. Assume that $f: R^{n \times n} \rightarrow R$ is strongly convex and continuously differentiable and $D$ is a compact set in $R^{n \times n}$. Let $\min _{X \in D}\left\|f^{\prime}(X)\right\|>0$. If $\psi(Z)=0$, then the point $Z$ is a solution to problem (30).

Proof. This is an obvious consequence of the following relations:

$$
0=\psi(Z) \leqslant P(Y) \leqslant\left\langle f^{\prime}(X), X-Y\right\rangle_{F},
$$

which are fulfilled for all $Y \in E_{f(Z)}(f)$ and $X \in D$.
Now we are ready to present an algorithm for solving problem (30). We also suppose that one can efficiently solve the problem of computing $\min _{X \in D}\left\langle f^{\prime}(X), X-Y\right\rangle_{F}$ for any given $Y \in R^{n \times n}$.

\section{Algorithm MIN}

Input. A strongly quasiconvex function $f$ and a compact set D.

Output. A solution $X$ to the minimization problem (30).

Step 1. Choose a feasible point $X^{0} \in D$. Set $k:=0$.

Step 2. Solve the following problem:

$$
\min _{Y \in E_{f\left(X_{k}\right)}(f)} P(Y) .
$$

Let $Y_{k}$ be a solution of this problem (i.e., $P\left(Y_{k}\right)=$ $\left.\min _{X \in D}\left\langle f^{\prime}(X), X-Y_{k}\right\rangle_{F}=\min _{Y \in E_{f\left(X_{k}\right)}(f)} P(Y)\right)$, and let $X_{k+1}$ realizes $P\left(Y_{k}\right)$ (i.e., $\left.\psi\left(X_{k}\right)=P\left(Y_{k}\right)=\left\langle f^{\prime}\left(X_{k+1}\right), X_{k+1}-Y_{k}\right\rangle_{F}\right)$.

Step 3. If $\psi\left(X_{k}\right)=0$ then output $X=X_{k}$ and terminate. Otherwise, let $k:=k+1$ and return Step 2.

The convergence of this algorithm is based on the following theorem.

Theorem 11. Assume that $f: R^{n \times n} \rightarrow R$ is strongly convex and continuously differentiable and $D$ is a compact set in $R^{n \times n}$. Let $\min _{X \in D}\left\|f^{\prime}(X)\right\|_{F}>0$. Then the sequence $\left\{X_{k}, k=\right.$ $0,1, \ldots\}$ generated by Algorithm MIN is a minimizing sequence for problem (30); that is,

$$
\lim _{k \rightarrow \infty} f\left(X_{k}\right)=\min _{X \in D} f(X),
$$

and every accumulation point of the sequence $\left\{X_{k}\right\}$ is a global minimizer of (30).

Proof. From the construction of $\left\{X_{k}\right\}$, we have $X_{k} \in D$ and $f\left(X_{k}\right) \geq f_{*}$ for all $k$, where $f_{*}=f\left(X_{*}\right)=\min _{X \in D} f(X)$. Clearly, $f^{\prime}\left(X_{*}\right) \neq 0$ by assumption. Also, note that for all $Y \in$ $E_{f\left(X_{k}\right)}(f)$ and $X \in D$, we have

$$
\begin{aligned}
\psi\left(X_{k}\right) & =\min _{Y \in E_{f\left(X_{k}\right)}(f)} \min _{X \in D}\left\langle f^{\prime}(X), X-Y\right\rangle_{F} \\
& \leqslant\left\langle f^{\prime}(X), X-Y\right\rangle_{F} \leqslant 0 .
\end{aligned}
$$

If there exists a $k$ such that $\psi\left(X_{k}\right)=0$ then, by Theorem 11, $X^{k}$ is a solution to problem (30) and in this case the proof is complete. Therefore, without loss of generality, we can assume that ignored $\psi\left(X_{k}\right)<0$ for all $k$ and prove the theorem by contradiction. If the assertion is false; that is, $X_{k}$ is not a minimizing sequence for problem (30), the following inequality holds:

$$
\lim _{k \rightarrow \infty} \inf f\left(X_{k}\right)>f_{*} .
$$


By the definition of $\psi\left(X_{k}\right)$ and Algorithm MIN, we have

$$
\begin{aligned}
p\left(Y_{k}\right) & =\psi\left(X_{k}\right) \\
& =\min _{Y \in E_{f\left(X_{k}\right)}(f)} \min _{X \in D}\left\langle f^{\prime}(X), X-Y\right\rangle_{F} \\
& =\left\langle f^{\prime}\left(X_{k+1}\right), X_{k+1}-Y_{k}\right\rangle_{F}
\end{aligned}
$$

and $f\left(Y_{k}\right)=f\left(X_{k}\right)$. The convexity of $f$ implies that

$$
\begin{aligned}
f\left(X_{k}\right)-f\left(X_{k+1}\right) & =f\left(Y_{k}\right)-f\left(X_{k+1}\right) \\
& \leqslant\left\langle f^{\prime}\left(X_{k+1}\right), Y_{k}-X_{k+1}\right\rangle_{F} \\
& =-\psi\left(X_{k}\right)>0 .
\end{aligned}
$$

Hence, we obtain $f\left(X_{k+1}\right)<f\left(X_{k}\right)$ for all $k$, and the sequence $\left\{f\left(X_{k}\right)\right\}$ is strictly decreasing. Since the sequence is bounded from below by $f_{*}$, it has a limit and satisfies

$$
\lim _{k \rightarrow \infty}\left(f\left(X_{k+1}\right)-f\left(X_{k}\right)\right)=0 .
$$

Then, from (49) and (50), we obtain

$$
\lim _{k \rightarrow \infty} \psi\left(X_{k}\right)=0 \text {. }
$$

From (51) we have $f\left(X_{k}\right)>f\left(X_{*}\right)$ for all $k$. Now define $v_{\alpha}$ as follows:

$$
V_{\alpha}=X_{*}+\alpha f^{\prime}\left(X_{*}\right), \quad \alpha>0 .
$$

Then, by the convexity of $f$, we have

$$
f\left(V_{\alpha}\right)-f\left(X_{*}\right) \geqslant\left\langle f^{\prime}\left(X_{*}\right), V_{\alpha}-X_{*}\right\rangle_{F}=\alpha\left\|f^{\prime}\left(X_{*}\right)\right\|^{2},
$$

which implies

$$
f\left(V_{\alpha}\right) \geq f\left(X_{*}\right)+\alpha\left\|f^{\prime}\left(X_{*}\right)\right\|^{2}>f\left(X_{*}\right), \quad \alpha>0 .
$$

Choose $\alpha=\alpha_{k}$ such that

$$
f\left(X_{*}\right)+\alpha_{k}\left\|f^{\prime}\left(X_{*}\right)\right\|^{2}>f\left(X_{k}\right)
$$

that is,

$$
\alpha_{k}>\frac{f\left(X_{k}\right)-f\left(X_{*}\right)}{\left\|f^{\prime}\left(X_{*}\right)\right\|^{2}}>0 .
$$

Define a function $h_{k}: R^{+} \rightarrow R$ as

$$
h_{k}(\alpha)=f\left(X_{*}+\alpha f^{\prime}\left(X_{*}\right)\right)-f\left(X_{k}\right),
$$

where $R^{+}=\{\alpha \in R \mid \alpha \geq 0\}$. It is clear that $h_{k}$ is continuous on $[0,+\infty)$. Note that $h_{k}\left(\alpha_{k}\right)>0$ and $h_{k}(0)<0$. Since $h_{k}$ is continuous, there exists a point $\overline{\alpha_{k}} \in\left(0, \alpha_{k}\right)$ such that $h_{k}\left(\overline{\alpha_{k}}\right)=0$; that is, $f\left(v_{\overline{\alpha_{k}}}\right)=f\left(x^{k}\right)$ and $v_{\overline{\alpha_{k}}}=x_{*}+\overline{\alpha_{k}} f^{\prime}\left(x_{*}\right)$. Also, note that

$$
\begin{aligned}
\psi\left(X_{k}\right) & =\min _{Y \in E_{f\left(X_{k}\right)}(f)} \min _{X \in D}\left\langle f^{\prime}(X), X-Y\right\rangle_{F} \\
& \leqslant\left\langle f^{\prime}\left(X_{*}\right), X_{*}-V_{\overline{\alpha_{k}}}\right\rangle_{F} \cdot
\end{aligned}
$$

Taking into account $V_{\overline{\alpha_{k}}}=X_{*}+\overline{\alpha_{k}} f^{\prime}\left(X_{*}\right)$, we have

$$
\begin{aligned}
-\psi\left(X_{k}\right) & \geqslant\left\langle f^{\prime}\left(X_{*}\right), V_{\overline{\alpha_{k}}}-X_{*}\right\rangle_{F} \\
& =\left\|f^{\prime}\left(X_{*}\right)\right\|\left\|V_{\overline{\alpha_{k}}}-X_{*}\right\| \\
& \geqslant \min _{X \in D}\left\|f^{\prime}(X)\right\|\left\|v_{\overline{\alpha_{k}}}-X_{*}\right\|>0 .
\end{aligned}
$$

Since $\lim _{k \rightarrow \infty} \psi\left(X_{k}\right)=0$, this implies

$$
\lim _{k \rightarrow \infty} V_{\overline{\alpha_{k}}}=X_{*}
$$

The continuity of $f$ on $R^{n \times n}$ yields

$$
\lim _{k \rightarrow \infty} f\left(X_{k}\right)=\lim _{k \rightarrow \infty} f\left(V_{\overline{\alpha_{k}}}\right)=f\left(X_{*}\right),
$$

which is a contradiction to (49).

Consequently, $\left\{X_{k}\right\}$ is a minimizing sequence for problem (30). Since $D$ is compact, we can always select the convergent subsequences $\left\{X_{k_{l}}\right\}$ from $\left\{X_{k}\right\}$ such that

$$
\lim _{l \rightarrow \infty} X_{k_{l}}=\bar{X} \in D
$$

Then together with (63), we obtain

$$
\lim _{l \rightarrow \infty} f\left(X_{k_{l}}\right)=f(\bar{X})=f_{*},
$$

which completes the proof.

\section{Numerical Experiments}

The proposed algorithm has been tested on the following numerical examples.

Problem 12.

$$
\begin{gathered}
\min _{X \in \mathbb{D}}\|A X-B X\|_{F}^{2}, \\
A=\left(\begin{array}{ll}
2 & 3 \\
3 & 4
\end{array}\right), \quad B=\left(\begin{array}{ll}
1 & 3 \\
2 & 4
\end{array}\right),
\end{gathered}
$$

where

$$
\mathbb{D}=\left\{\begin{array}{l}
\left\langle A_{1} X, X\right\rangle_{F}+\left\langle A_{2}, X\right\rangle_{F} \leqslant 0 \\
\left\langle A_{2} X, X\right\rangle_{F}+\left\langle A_{1}, X\right\rangle_{F} \leqslant 0 \\
A_{1}=\left(\begin{array}{cc}
-1 & 2 \\
3 & 4
\end{array}\right), \quad A_{2}=\left(\begin{array}{ll}
2 & 3 \\
1 & 4
\end{array}\right) \\
X \geqslant 0 .
\end{array}\right.
$$

The global solution is

$$
X^{*}=\left(\begin{array}{ll}
4 & 4 \\
2 & 5
\end{array}\right) \text {. }
$$

Problem 13.

$$
\begin{aligned}
& \min _{X \in D}\|X\|_{F}^{2} \text { subject to } \mathbb{D}= \\
&=\left\{X \in \mathbb{R}^{n \times n} \mid \underline{X}=\left(\begin{array}{ll}
2 & 3 \\
4 & 1
\end{array}\right) \leqslant X \leqslant \bar{X} .\right. \\
&\left.=\left(\begin{array}{ll}
7 & 5 \\
8 & 9
\end{array}\right), X \geqslant 0\right\}
\end{aligned}
$$


The global solution is

$$
X^{*}=\left(\begin{array}{ll}
4 & 3 \\
8 & 9
\end{array}\right)
$$

\section{References}

[1] A. Bouhamidi, R. Enkhbat, and K. Jbilou, "Semidefinite concave programming," Mongolian Mathematical Journal, pp. 37-47, 2012.

[2] R. T. Rockafellar, Convex Analysis, Princeton University Press, Princeton, NJ, USA, 1970.

[3] R. Enkhbat and T. Ibaraki, "On the maximization and minimization of a quasiconvex function," Journal of Nonlinear and Convex Analysis, pp. 43-76, 2011. 


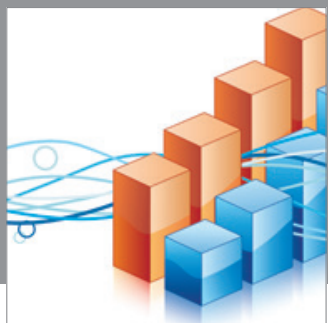

Advances in

Operations Research

mansans

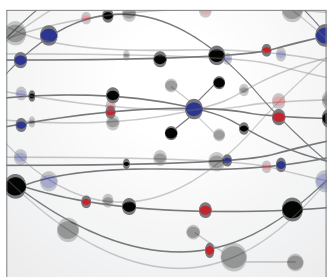

The Scientific World Journal
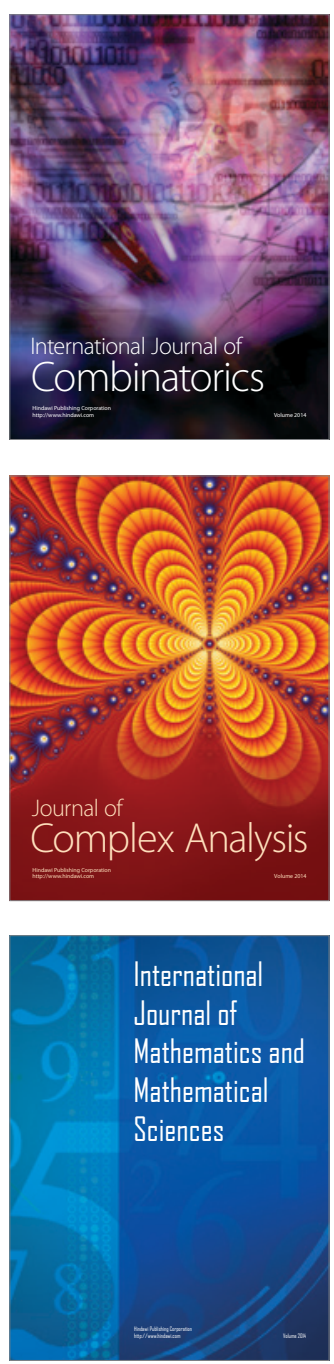
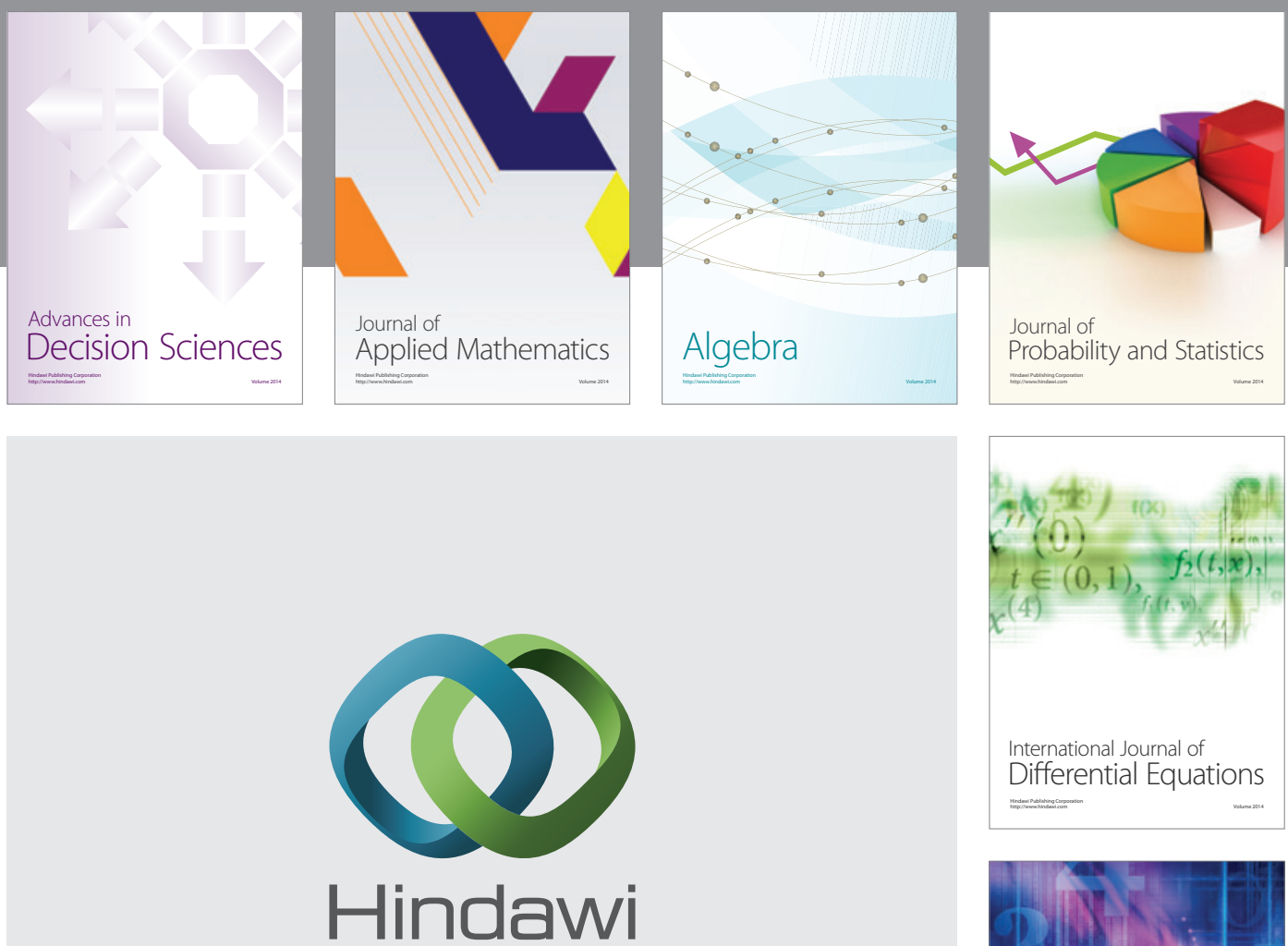

Submit your manuscripts at http://www.hindawi.com
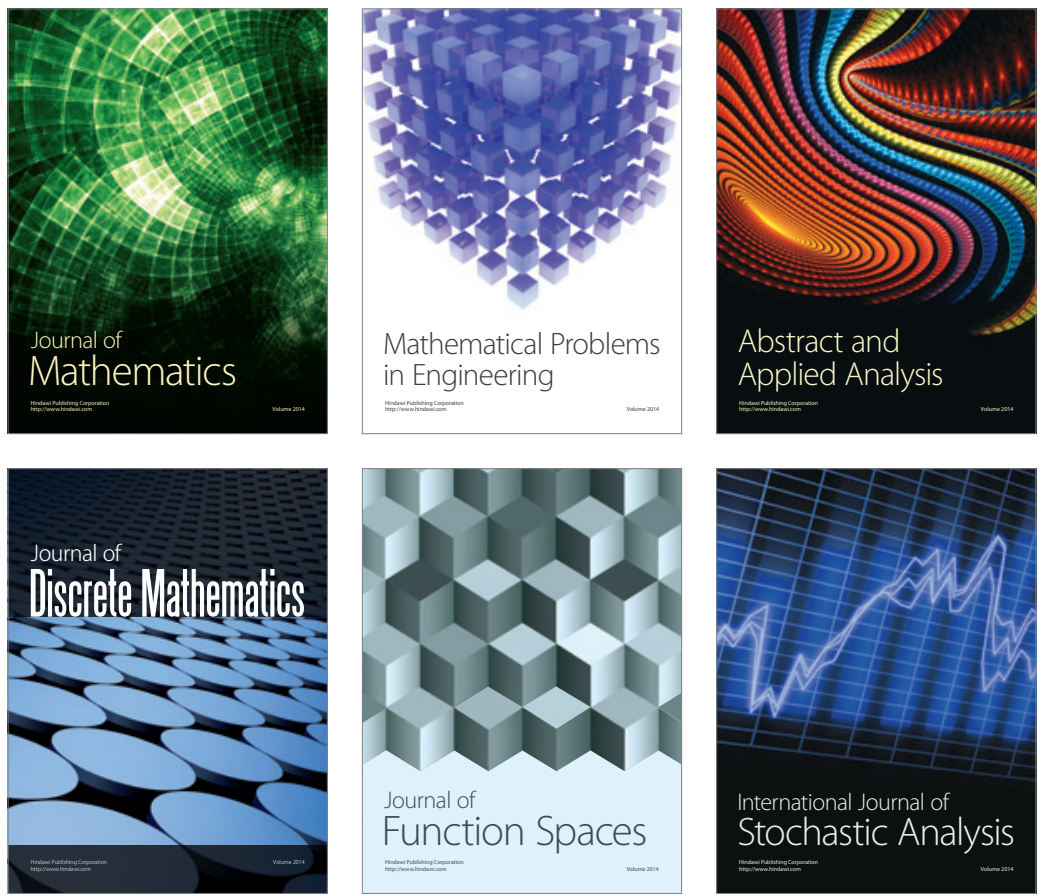

Journal of

Function Spaces

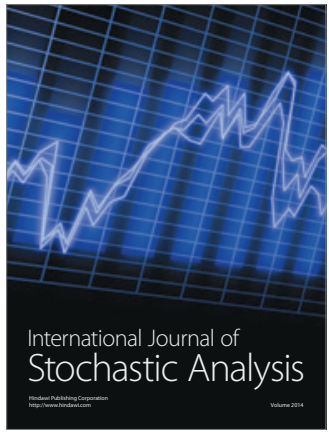

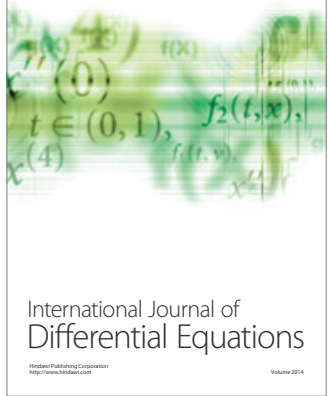
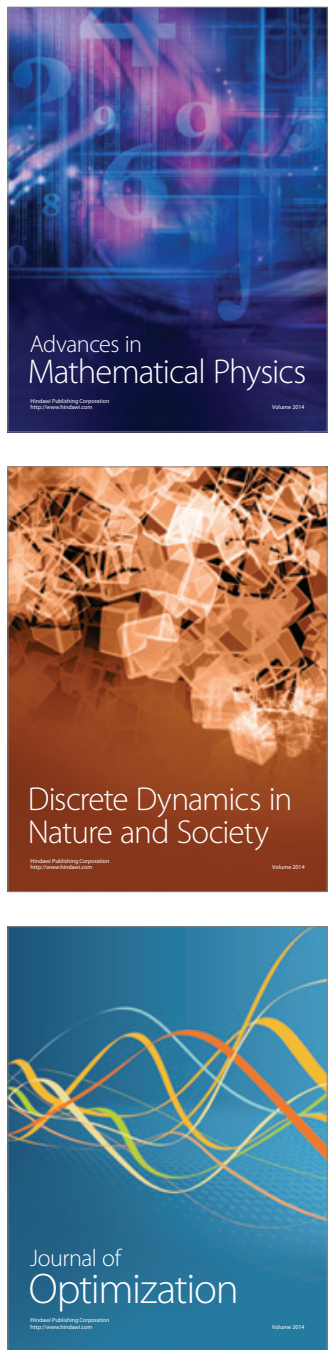